\title{
Agôn
}

Revue des arts de la scène

Critiques | Saison 2009-2010

\section{De la naissance à la connaissance}

À propos de Forêts, pièce écrite et mise en scène par Wajdi Mouawad

\section{Wendy Viste}

\section{(2) OpenEdition}

1 Journals

Édition électronique

URL : http://journals.openedition.org/agon/575

DOI : 10.4000/agon.575

ISSN : 1961-8581

Éditeur

Association Agôn

Référence électronique

Wendy Viste, « De la naissance à la connaissance », Agôn [En ligne], Critiques, mis en ligne le 05 février 2008, consulté le 23 septembre 2020. URL : http://journals.openedition.org/agon/575 ; DOI : https:// doi.org/10.4000/agon.575

Ce document a été généré automatiquement le 23 septembre 2020

Association Agôn et les auteurs des articles 


\title{
De la naissance à la connaissance
}

\author{
À propos de Forêts, pièce écrite et mise en scène par Wajdi Mouawad
}

\author{
Wendy Viste
}

1 Assister à un spectacle écrit et mis en scène par Wajdi Mouawad, c'est comme plonger entièrement sa tête dans un livre et y mettre toute son énergie jusqu'à la résolution finale. Le théâtre qu'il écrit raconte des histoires à la manière d'un roman, avec toutes les qualités que cela requiert. Ses pièces ressemblent à des romans adaptés pour la scène théâtrale, elles ont même des qualités de scènes de cinéma. L'enchaînement des lieux, les changements d'époque, et le mouvement qui prend forme sur scène tel celui d'une caméra, confèrent à ses spectacles ce caractère filmique. Cela tient sûrement au fait que le texte est écrit après une session de travail et d'improvisation entre les comédiens et l'auteur. Cette méthode valait déjà pour ses deux précédentes mises en scène, Littoral et Incendies, dans lesquelles il amorçait un travail autour de l'enquête. Il propose un troisième volet avec Forêts, spectacle créé en 2006, joué la saison dernière et repris pour la saison 2007-2008, au théâtre des Célestins du 3 au 12 avril 2008. Ce travail, il l'achèvera avec une quatrième création qui aura le nom cette fois de Ciels. Une quatrième partie qui viendra renverser tout le travail et les propos des trois dernières, à savoir, l'importance de connaître ses origines, le passé et la promesse. "Sans cette contradiction qui arriverait comme un point d'orgue, final, la violence ne serait pas entière. ", c'est ce que Wajdi Mouawad écrit dans une note du 25 avril 2006, et qui ouvre le livre de Forêts.

LA marque du corps. Cette violence dont il parle, est bien présente dans le spectacle de Forêts, sous un de ses nombreux visages. Elle se trouve surtout dans les pans d'histoire à travers l'Histoire, que Mouawad a décidé de raconter. Mises à part quelques scènes qui peuvent déranger, les images créées sont fortes, mais ne sont pas violentes. Cette sensation tient peut-être au fait que dans le théatre contemporain, il est de plus en plus fréquent d'assister à des scènes où les corps se déchaînent, et où l'on ressent une certaine souffrance à la vue de corps qui sont montrés nus, dans des positions dévoilant une monstruosité. Dans Forêts, les corps sont engagés, dévoilés, mais ne provoquent pas de souffrance pour celui qui les regarde. C'est plus ce qu'ils racontent 
que comment ils le racontent qui est bouleversant. La poésie des dialogues se transmet aux images et permet d'adoucir le degré de violence des histoires qui se succèdent. L'histoire et la mise en scène sont claires, et font en sorte de ne pas perdre l'attention du public malgré des entrelacements incessants. Les superpositions spatio-temporelles ne laissent part à aucune maladresse, et transforment la scène du théâtre en un arbre généalogique vivant. Le personnage d'Aimée qui ouvre la pièce avec un monologue est attachante, et cela est important pour tout le reste de l'histoire. On se sent investi dans l'épreuve à laquelle elle se trouve confrontée. Et c'est bien de corps et de marques sur le corps, comme les tatouages de certains personnages, qu'il s'agit pour servir le relais et le lien entre tous, et non pas de sang. De plus, ce début, ancré dans notre époque, facilite la rétrospection dans la descendance des personnages. Le réalisme des personnages appartenant au vingtième siècle, c'est-à-dire Aimée, Loup, Douglas Dupontel, Baptiste, Luce et Achille, permettent de prendre le propos de l'ensemble de la pièce au sérieux, et non pas seulement comme des légendes mises en scène.

LA marque de l'histoire. En dehors de quelques passages qui laissent perplexes et n'atteignent pas toujours le degré d'émotion voulue, une chose indéniable est l'intelligence et la précision de la mise en scène. Une scénographie simple et uniforme qui s'accorde avec tous les lieux représentés et les situations jouées. De manière générale, les lieux de la pièce sont des endroits plutôt froids, comme l'hôpital. Des endroits sauvages, ou plus exactement, des endroits où la sauvagerie humaine a pris le dessus sur la civilisation, comme la forêt.

4 La structure en place sur scène offre un espace carré constitué de trois cloisons à cour, à jardin et en fond de scène, dans les tons bleu-gris. Grâce au jeu des lumières, les couleurs changent et la scène n'est plus qu'un espace noir, ou bien ocre. En fonction de l'éclairage qui lui est donné, la cloison en fond de scène prend l'apparence d'un mur ou d'un voile, à travers lequel la scène de meurtre dans la grotte sera représentée en jeu d'ombre pour renvoyer l'apparence du monstre qui y vit. Ainsi on assiste au meurtre de façon détournée, en évitant le ridicule qu'impose la difficulté de ce genre de scènes. Cette difficulté, Wajdi Mouawad a su l'éviter tout au long du spectacle en assumant totalement le fait qu'on soit au théâtre. Il y a cette table en bois sans cesse manipulable, et manipulée, utilisée, par exemple, au moment de l'accouchement d'Aimée. On en voit sortir, par le trou qui se trouve en son centre, le corps de Loup avec l'âge qu'elle a à l'époque d'aujourd'hui, c'est-à-dire seize ans. L'attribution de différentes fonctions à un même objet, c'est aussi et surtout en cela que réside l'une des fonctions théâtrales, une ouverture sur l'imaginaire. Au final, il y a peu d'objets sur la scène, peuplée la plupart du temps des personnages et de leurs voyages dans le temps et l'espace. Seule la table en bois sert de lien entre tous. La table autour de laquelle on partage un repas avec ses amis, la table autour de laquelle on discute, se dispute, celle sur laquelle on accouche, on se fait opérer, et celle finalement sur laquelle notre corps froid se retrouve, et autour de laquelle nos proches viennent pleurer et se recueillir une dernière fois. Tout cela est dans la pièce, la boucle, le cycle, la descendance. Les gens naissent, vivent et meurent, tandis que les objets, eux, perdurent à travers les années. L'être humain est une denrée périssable qui une fois morte ne laisse comme trace que son passé.

LA marque du temps. Le passé et le présent, Wajdi Mouawad les réunit dans le même espace afin de trouver les clés qui ouvriront les portes d'un avenir serein. L'écriture et 
la mise en scène se recoupent de telle manière que certains personnages semblent se parler d'une génération à une autre. Les fantômes qui hantent les esprits de chacun prennent vie et évoluent sur le même plan, et tout en renforçant l'écart qu'il y a entre eux, ces images scéniques les rendent proches. Elles rendent aussi possible une rencontre souvent espérée qui n'a jamais pu avoir lieu. Cet effet de mélange de plans et de flash-backs, plus souvent utilisés au cinéma, prennent ici toute leur dimension en révélant avec d'autant plus de force les gâchis et erreurs perpétrés, comme un héritage d'enfants à parents. Des personnages, des noms de personnages apparaissent, disparaissent puis réapparaissent venant mettre de l'ordre dans l'enquête et dans l'esprit du spectateur. La trame n'est pas facile à suivre, ou bien, facile à faire suivre et c'est cela qui fait la réussite du spectacle. L'organisation est remarquable et cependant le spectacle dans son entier n'égale pas toujours ce degré de qualité. Ce qui au commencement du spectacle était touchant devient pesant à mesure qu'on remonte le cours des générations. Et ce qui était émouvant devient malheureusement aberrant à mesure que les questions trouvent leur réponse. Peut-être manque-t-il une once de rationalité dans ce qui relie les vécus des personnages. Il est évident que la tournure que prennent les événements sert le propos de l'auteur, à savoir que la mémoire commune est plus forte que les liens du sang. La fiction amène parfois les créateurs à en faire trop et à se laisser emporter dans un tourbillon où chaque scène se justifie au moment où elle intervient dans l'histoire car s'installe un effet de surenchère.

6

LA marque de l'auteur-metteur en scène. L'envie de reconnaitre et de saluer la qualité et l'énergie du travail effectués par l'auteur et les comédiens n'empêche pas quelques réserves. Les malédictions qui se succèdent forment un tout qui concentre trop d'horreurs et d'événements incroyables. Plus on découvre la vérité, moins on arrive à y croire et de ce fait, à être totalement ému. D'incestes en viol, d'avortements en meurtre, aucune action tragique n'est épargnée. De plus, les acteurs jouent dans une direction qui rend les scènes encore plus larmoyantes et dramatiques. Le texte est déjà assez fort et dirigé vers la tragédie pour ne pas en rajouter encore plus. Quelques scènes se font plus ennuyeuses que d'autres. Il arrive un moment dans le spectacle où l'on aurait besoin d'un peu de temps pour tout remettre en place, heureusement cela arrive grâce au paléontologue, mais n'empêche pas le fait d'avoir décroché durant quelques minutes.

7 La direction des acteurs alourdit les caractères dramatique et tragique inhérentsau texte, et ne permet pas de recul pour le spectateur qui est baladé d'une époque à une autre et d'une épreuve à une autre. Un trop plein de sentimentalisme découle de ce manque de liberté laissé au spectateur. L'image de fin en est un exemple frappant, et soit on salue l'idée des pétales de rose, soit on rejette d'être autant soumis à l'émotion. Ces débordements et cette volonté de trop et de toujours plus, ne font cependant pas de Forêts un spectacle ennuyeux et sans intérêt. Wajdi Mouawad a réussi un travail qui laisse des traces sur la scène théâtrale et montre la perception d'un auteur aux multiples facettes. 


\section{AUTEUR}

WENDY VISTE

Étudiante en deuxième année de Licence Arts du spectacle, Université Lumière - Lyon 2 